\title{
Hemisensory Deficit in a Patient with Creutzfeldt-Jakob Disease
}

Dr. I. Kothbauer-Margreiter, Neurologische Klinik, Inselspital, CH-3010 Bern (Switzerland)

Creutzfeldt-Jakob disease (CJD) is a human prion disease causing degeneration of the central nervous system [1]. The characteristic clinical features of the disorder are neuropsychological deficits progressing to dementia, myoclonus and cerebellar dysfunction. Less frequent symptoms include extrapyramidal and pyramidal signs,
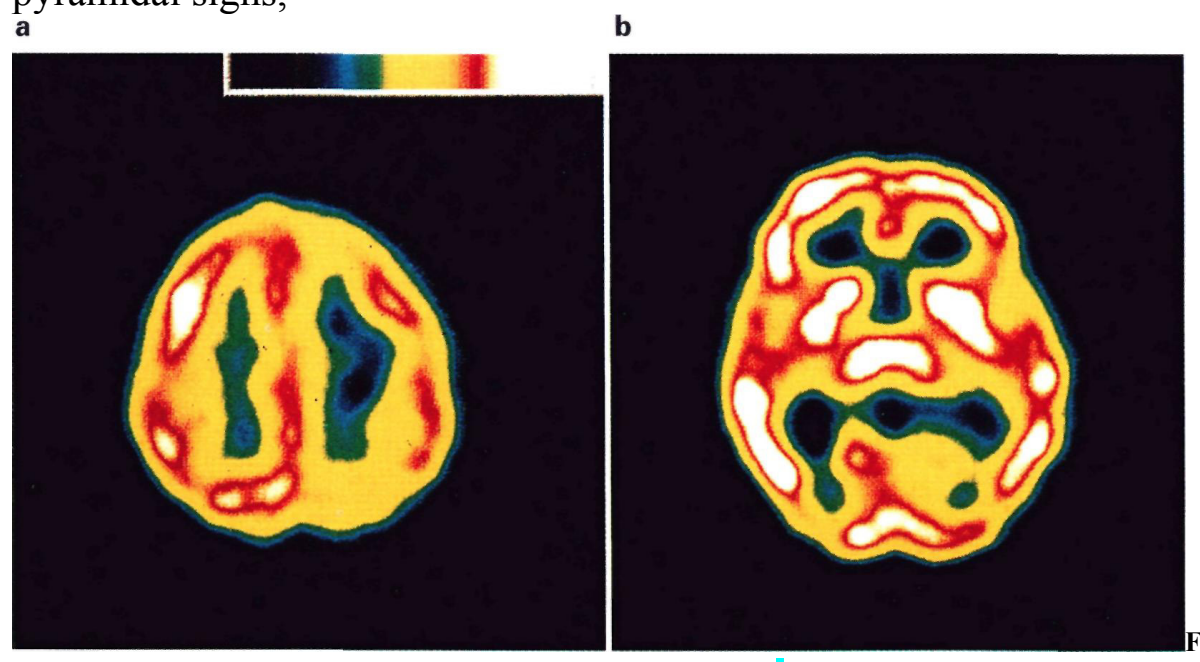

racer uptake of thalamic nuclei of both sides (b). Courtesy: Dr. C. Als, Department of Nuclear Medicine, Bern.

108

visual impairment and epileptic seizures [2]. To our knowledge the occurrence of hemisensory deficits has not yet been described. We report a patient with autopsy-proven CJD who presented with a severe unilateral pseudothalamic sensory deficit in addition to neuropsychological and cerebellar dysfunction. This 49-year-old man was admitted with gait ataxia and dysarthria of sudden onset 5 weeks before. There was a 5 months' history of short-term memory disturbance and emotional lability. The previous medical history was unremarkable. The patient took no medications. Neurological examination disclosed disorientation to time and place, mild constructive apraxia, cerebellar signs including oculomotor dysfunction, hypotonia and postural imbalance, and myoclonic jerks. Furthermore, a faciobrachiocrural hemisensory deficit on the right side with severe impairment of pinprick, touch, temperature, vibration, position sense and stereognosis was found. Furthermore, intermittent choreo-athetotic movements of the right hand were seen. Blood, urine and cerebrospinal fluid were normal. Cerebral magnetic resonance imaging (MRI) was normal. Two single photon emission computed tomography (SPECT) examinations of the brain after intravenous injection of Tc-99m hexamethyl-propyleneamineoxime (HMPAO) showed a decreased tracer uptake in the entire left hemisphere sparing the basal ganglia including thalamic nuclei of both sides (fig. 1). Initial EEG documented a left hemispheric focal theta-delta activity with periodic triphasic discharges. Later EEGs delineated a continuous spread of slow activity to the contralateral hemisphere. Finally, triphasic rhythmic discharges were present in both hemispheres.

The clinical course initially showed a fluctuant symptomatology with a significant temporary improvement of ataxia, speech and mental status. Later on, dementia rapidly progressed and the patient's status deteriorated. He died within 10 weeks after the onset of symptoms. At autopsy a spongiform encephalopathy of the grey matter of both cerebral hemispheres without involvement of the basal ganglia or the thalami was found. Cerebellum and brain stem were only slightly affected while the white matter was normal.

The occurrence of lateralized neurological deficits described in our patient is well known in the initial stages of CJD [3]. However, to our knowledge hemisensory deficits due to CJD have not yet been reported

[4]. The deficit in this patient consisted of a severe impairment of all modalities of sensation in the right hemibody as typically observed in thalamic, more rarely in subcortical or cortical lesions [5,6].

The association with mild constructive apraxia in our patient pointed to a parietal lobe dysfunction. This assumption was underlined by pathological SPECT, initial EEG and autopsy. 